\section{Veterinaria México OA}

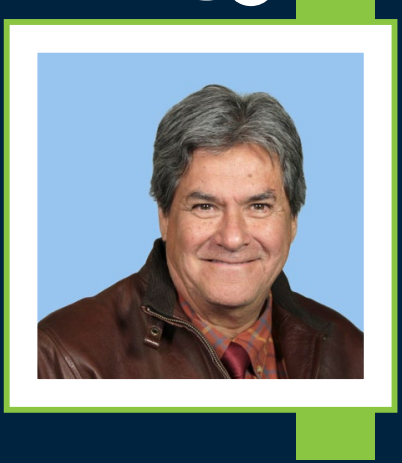

\section{Carlos Galina Hidalgo}

El Dr. Carlos Galina Hidalgo ha sido docente del Departamento de Reproducción Animal de la Facultad de Medicina Veterinaria y Zootecnia de la Universidad Nacional Autónoma de México desde 1973. Su contribución en la investigación sobre los problemas en la detección de los signos de estro en el ganado tropical ha permitido una mejor difusión de las técnicas de inseminación artificial y transferencia de embriones en estos animales, así como del uso de toros en programas reproductivos bajo condiciones tropicales. Es investigador emérito del Sistema Nacional de Investigadores y en el 2009 fue nombrado profesor emérito en la Universidad Nacional Autónoma de México debido a su destacada trayectoria académica.

* Profesor Emérito de la Universidad Nacional Autónoma de México

IFacultad de Medicina Veterinaria y Zootecnia, Universidad Nacional Autónoma de México, México

${ }^{2}$ Centro Universitario de Investigaciones Bibliotecológicas, Universidad Nacional Autónoma de México, México

Autor para correspondencia: Correo electrónico:

cgalina@unam.mx

The journal "Veterinaria México" as a communication channel for research in veterinary science and zootechnics

D'Alessandro E, Cárdenas P, Russell JM, Galina CS.

La revista Veterinaria México como medio de difusión de la investigación en ciencias veterinarias y zootécnicas. Veterinaria México. 2000;31(3):261-6.

\section{Publicado: 2020-09-30}

Información y declaraciones adicionales en la página 11

CD Derechos de autor:

Enrique d'Alessandro et al. 2020

$$
\text { acceso abierto } 0 \text { (c) (i) }
$$

Distribuido bajo una Licencia Creative Commons Atribución 4.0 Internacional (CC-BY 4.0)
Veterinaria

\section{La revista Veterinaria México como medio de difusión de la investigación en ciencias veterinarias y zootécnicas}

Enrique D'Alessandro'

Paula Cárdenas ${ }^{1}$

Jane M. Russell ${ }^{2}$

Carlos S. Galina*1

\section{Abstract}

The Mexican research journal Veterinaria Mexico has been published without interruption by the Faculty of Veterinary Medicine and Zootechnics (FMVZ) at the National Autonomous University Of Mexico (UNAM) since 1970. Analysis of the 518 studies published in the 40 issues of the journal from 1989 to 1998 showed that research articles were the most frequent type of contribution to the journal representing $60 \%$. Subject areas of the contributions were mainly pathology, followed by reproduction, parasitology and pharmacology, corresponding to $47 \%$ of the total. Bovine were the most studied species appearing in almost a quarter of the papers followed by poultry, pigs and dogs, all with approximately 10\% each. Authors from the FMVZ participated in only $33 \%$ of papers indicating the importance of this journal as a vehicle for the publication of results from other national and international institutions. One hundred and eighty contributions were found for the FMVZ published in co-authorship with other institutions within the UNAM or with other national institutions, whereas contributions in international collaboration of the FMVZ amounted to 19. Finally, foreign institutions were responsible for 67 contributions without the FMVZ, and national institutions for 228 .

Keywords: Mexico; Veterinary medicine; Animal production; Journals; Research; Contribution

\section{Cite this as:}

D’Alessandro E, Cárdenas P, Russell JM, Galina CS. La revista Veterinaria México como medio de difusión de la investigación en ciencias veterinarias y zootécnicas. Veterinaria México OA. 2020:7(3). doi:10.22201/fmvz.24486760e.2020.3.921. 


\section{Resumen}

La Facultad de Medicina Veterinaria y Zootecnia (FMVZ) de la Universidad Nacional Autónoma de México (UNAM), ha publicado desde 1970, ininterrumpidamente la revista científica Veterinaria México. En el presente estudio se analizaron 518 artículos en 40 números publicados desde 1989 hasta 1998, los trabajos más frecuentes fueron artículos científicos (60\%). Con respecto al área de trabajo, patología fue la más frecuente, seguida por reproducción, parasitología y farmacología, que representan globalmente el 47\% del total. Entre las especies más estudiadas, bovinos representó casi $25 \%$ de los trabajos, seguido de aves, cerdos y perros con aproximadamente $10 \%$ cada una. El grupo de autores en donde sólo participa la FMVZ representa 33\% (250) del total, esto último indica la importancia de esta revista como medio para la publicación de trabajos procedentes de otras instituciones, tanto nacionales como extranjeras. Asimismo, el grupo de otras instituciones nacionales que participan en coautoría con la FMVZ tuvieron 26\% (199) de participación de estos últimos, 180 fueron nacionales mientras que las extranjeras tuvieron 19. Por último, las contribuciones de instituciones sin la participación de autores de la FMVZ fue del 38\% (295), de los cuales 228 correspondió a instituciones nacionales y 67 extranjeras

Palabras clave: México; Medicina veterinaria; Producción animal; Revistas; Investigaclón; Contribución 
Las revistas científicas representan el mejor medio de comunicación y difusión de la ciencia. ${ }^{l}$ En ellas, los investigadores tienen oportunidad de publicar los resultados de sus investigaciones. En virtud de que se trata de revistas especializadas, destinadas a lectores también especializados, el investigador tiene la seguridad de que su trabajo tendrá una acertada difusión, así como de que este último no pasará inadvertido para la comunidad científica. ${ }^{2}$

Las revistas de ciencias veterinarias editadas en América Latina difunden la investigación que se realiza, especialmente en los campos de medicina aplicada, cuya difusión de resultados se hace principalmente por medio de las revistas editadas localmente. En este contexto, estas publicaciones permiten que se conozca el trabajo científico que se realiza en la región.

La revista Veterinaria México constituye una publicación trimestral de la Facultad de Medicina Veterinaria y Zootecnia (FMVZ) de la Universidad Nacional Autónoma de México (UNAM) ${ }^{3}$ El objetivo de la revista consiste en difundir en los ámbitos nacional e internacional, los más recientes avances en investigación animal. ${ }^{4}$

El compromiso de la FMVZ es crear, desarrollar e impulsar publicaciones científicas con el propósito de fortalecer el liderazgo de la UNAM en la formación de investigadores de calidad, con el propósito de reafirmar el carácter científico nacional y dar lineamientos a otras instituciones en la formación de investigadores de excelencia. ${ }^{2}$

Fundada en 1970, Veterinaria México cumplió 30 años de aparición ininterrumpida; su trascendencia es notable, pues se incluye en importantes servicios especializados de información: Index Veterinarius, Veterinary Bulletin, Chemical Abstracts, Biology Abstracts, Focus on: Veterinary Science and Medicine, entre muchos otros. Asimismo, a partir de 1997 está disponible en el disco compacto Artemisa, que contiene en texto completo, los artículos que se publican en las revistas biomédicas mexicanas de mayor calidad. ${ }^{3}$ En 1997 Veterinaria México fue aceptada en el Índice de Revistas Mexicanas de Investigación Científica y Tecnológica, del Consejo Nacional de Ciencia y Tecnología (Conacyt), cuyos criterios de evaluación no sólo se refieren a la calidad del contenido, sino también a la inclusión en índices internacionales y a la periodicidad y regularidad de la publicación. ${ }^{3,5}$ Los criterios de evaluación utilizados por el comité de revistas científicas del Conacyt establecen que los artículos incluidos en la revista sean producto de investigaciones originales, en las que incorporen material de todo tipo, como información general, ensayos, reseñas o traducciones, pero siempre en una proporción mínima y equilibrada. Por otro lado, la calidad del contenido de la revista debe estar respaldada por un consejo editorial, integrado por investigadores de prestigio, que de preferencia tenga una representación y reconocimiento institucional e internacional. ${ }^{4}$

Cabe mencionar que también se encuentra dentro del Índice de Conacyt la revista Técnica Pecuaria, que es editada en forma cuatrimestral por el Instituto de Investigaciones Forestales, Agrícolas y Pecuarias, de la Secretaría de Agricultura, Ganadería y Desarrollo Rural (SAGAR) para difundir, al igual que Veterinaria México, la investigación en ciencias veterinarias; éstas son las dos únicas revistas de ciencias aplicadas que se encuentran en el Índice del Conacyt. ${ }^{5}$ 
Una revisión de los artículos publicados en Veterinaria México de 1985 a 1992 reveló que 55.3\% de éstos corresponde a autores de la propia FMVZ; de igual manera $4.2 \%$ de aquéllos procede de autores de dependencias extranjeras. Esto último indica que la revista representa un medio de difusión importante para otras instituciones nacionales, sin desconocer su participación en la comunicación de trabajos originales provenientes de instituciones de otros países. ${ }^{2}$

Con el propósito de situar la investigación realizada por los especialistas de la FMVZ en años recientes, así como con el afán de ofrecer un panorama general de las investigaciones del área a la comunidad científica nacional, en el presente trabajo se revisaron 40 números de Veterinaria México publicados entre 1989 y 1998. En primera instancia, se procedió a analizar cada uno de los artículos y a clasificarlos de acuerdo con el tipo de estudio, el área, o la especie a tratar, así como las instituciones que colaboraron en dichos proyectos, ya sean de México o el extranjero.

Para desarrollar el presente trabajo, se utilizaron 40 números de la revista Veterinaria México, publicados en un lapso de 10 años, 1989 a 1998 (volúmenes 20 al 29). A lo largo de este tiempo, la revista cambió en unas ocasiones la nomenclatura para la clasificación de los trabajos. Con el fin de facilitar la consulta, los autores no consideraron tales diferencias y decidieron utilizar la siguiente clasificación de trabajos, por considerarla conveniente para la comprensión del presente estudio: Memoria científica; Nota informativa; Artículo recapitulativo (a partir del volumen 28, número 4, 1997, cambiaron a Artículo científico, Nota de investigación, Artículo de revisión, respectivamente); Artículo por invitación, esta modalidad se publicó sólo hasta 1995; a sugerencia de Conacyt, se eliminó.

Se clasificaron las contribuciones publicadas de acuerdo con las áreas tratadas y las especies estudiadas.

Un gran número de los trabajos que se publican en la revista son investigaciones realizadas en coautoría con investigadores que trabajan en instituciones de diferentes sectores (educativos, gubernamentales, privados, de investigación, organismos internacionales, como la FAO, Greenpeace) y con otras instituciones o dependencias que forman parte de la UNAM.

Por lo tanto, se ha señalado la procedencia de las contribuciones y se ha dado el crédito correspondiente a cada uno de los sectores. De esta forma, se especifican las contribuciones provenientes de: la FMVZ (en este caso se especifican los departamentos involucrados); la FMVZ en colaboración con otras instituciones; colaboradores ajenos a la FMVZ.

Además, las contribuciones han sido clasificadas en dos ámbitos: nacional y extranjero, según la procedencia de las instituciones que intervinieron en su realización.

Con fines de esta revisión, se han denominado contribuciones a las participaciones individuales de los trabajos publicados por los autores de los diferentes departamentos, instituciones y sectores

A partir del análisis de 518 trabajos publicados en 40 números de la revista Veterinaria México, volúmenes 20 al 29, correspondientes al periodo 1989 hasta 1998, se observó que los trabajos más frecuentemente publicados son los que se 
Cuadro 1. Categorias de los trabajos encontrados en las publicaciones de la revista durante el período de 1989 a 1998

\begin{tabular}{|l|c|c|c|c|c|c|c|c|c|c|c|}
\hline \multicolumn{1}{|c|}{ Tipo de publicación } & $\mathbf{1 9 8 9}$ & $\mathbf{1 9 9 0}$ & $\mathbf{1 9 9 1}$ & $\mathbf{1 9 9 2}$ & $\mathbf{1 9 9 3}$ & $\mathbf{1 9 9 4}$ & $\mathbf{1 9 9 5}$ & $\mathbf{1 9 9 6}$ & $\mathbf{1 9 9 7}$ & $\mathbf{1 9 9 8}$ & TOTAL \\
\hline Memoria Científica & 24 & 31 & 27 & 27 & 28 & 31 & 34 & 35 & 34 & 37 & 308 \\
\hline Nota Informativa & 7 & 9 & 13 & 18 & 17 & 21 & 15 & 19 & 19 & 11 & 149 \\
\hline Caso Clínico & 0 & 2 & 3 & 4 & 5 & 6 & 3 & 0 & 0 & 0 & $23^{\S}$ \\
\hline Análisis Bibliométrico & 0 & 0 & 0 & 0 & 0 & 1 & 0 & 0 & 0 & 0 & $1^{\$}$ \\
\hline Estudio Recapitulativo & 6 & 2 & 0 & 2 & 1 & 0 & 4 & 5 & 5 & 8 & 33 \\
\hline Artículo por invitación & & 2 & 4 & 4 & 6 & 7 & 5 & 0 & 0 & 0 & 28 \\
\hline Total & 37 & 44 & 44 & 51 & 52 & 59 & 58 & 59 & 58 & 56 & 518 \\
\hline
\end{tabular}

$\mathbb{\$}$ Se encuentran contabilizadas dentro de la Nota Informativa.

catalogan como "Memoria científica" (308 trabajos). Se destaca un incremento significativo de siete trabajos en los años de 1989 a 1990 y de 1993 a 1994. De 1994 hasta 1998, la revista se mantiene con un promedio anual de 58 trabajos. La siguiente contribución en importancia fue la "Nota informativa", con 149 trabajos, donde se incluyen casos clínicos y el análisis bibliométrico a partir de 1996 (Cuadro 1).

Con respecto al área en la que se suscriben los trabajos, Patología constituye la más frecuente, siguen en orden descendente Reproducción, Parasitología y Farmacología, con un global de 293 trabajos, equivalentes al 47\% del total de trabajos publicados (Cuadro 2). En cuanto a las especies, los bovinos son los más estudiados, seguidos en orden descendente por aves, cerdos y perros. Estas especies están presentes en 286 trabajos, que representa 61\% del total (Figura 1).

La Facultad de Medicina Veterinaria y Zootecnia de la UNAM participó con un total de 250 contribuciones en 172 trabajos. Los departamentos de Cerdos, Aves y Rumiantes tienen el mayor número de participaciones, después están Patología y Farmacología-Fisioiogía (Figura 2).

En coautoría con otras instituciones, tanto nacionales $(\mathrm{n}=180)$ como extranjeras ( $\mathrm{n}=19)$, la FMVZ tuvo 199 contribuciones. Las instituciones que participaron provienen de los sectores educativo, gubernamental, privado, investigación, organismos internacionales y de otras instituciones dentro de la UNAM; en este sentido, las áreas de educación e investigación tuvieron mayor número de colaboraciones con la FMVZ (Figura 3).

Los artículos en donde la FMVZ no participó, las cubrieron distintas instituciones nacionales $(n=228)$ y extranjeras $(n=67)$, también aquí los sectores educativo y de investigación, fueron los más frecuentados (Figura 4).

El mayor número de contribuciones (449) correspondió a la FMVZ, incluidas las individuales y las de coautoría con otros sectores, contra 295 contribuciones externas (Figura 5).

Por último, se compararon los sectores con los que la FMVZ tuvo alguna colaboración, y los sectores donde no tuvo ninguna participación. El sector educativo en coautoría con la FMVZ fue el más representativo (59 trabajos) (Figura 6). 
Cuadro 2. Áreas de los trabajos encontrados en Veterinaria México

\begin{tabular}{|l|c|c|c|c|c|c|c|c|c|c|c|}
\hline & $\mathbf{1 9 8 9}$ & $\mathbf{1 9 9 0}$ & $\mathbf{1 9 9 1}$ & $\mathbf{1 9 9 2}$ & $\mathbf{1 9 9 3}$ & $\mathbf{1 9 9 4}$ & $\mathbf{1 9 9 5}$ & $\mathbf{1 9 9 6}$ & $\mathbf{1 9 9 7}$ & $\mathbf{1 9 9 8}$ & TOTAL \\
\hline Patología & 4 & 5 & 10 & 11 & 12 & 6 & 7 & 5 & 14 & 6 & 80 \\
\hline Reproducción & 11 & 5 & 5 & 7 & 8 & 6 & 8 & 10 & 7 & 6 & 73 \\
\hline Parasitología & 3 & 5 & 9 & 7 & 8 & 9 & 8 & 6 & 10 & 6 & 71 \\
\hline Farmacología & 7 & 7 & 9 & 8 & 5 & 6 & 13 & 6 & 2 & 6 & 69 \\
\hline Inmunología & 2 & 2 & 5 & 10 & 6 & 3 & 5 & 5 & 8 & 6 & 52 \\
\hline Bacteriología & 3 & 5 & 6 & 6 & 3 & 4 & 8 & 8 & 4 & 2 & 49 \\
\hline Nutrición & 4 & 4 & 3 & 2 & 5 & 5 & 5 & 6 & 1 & 9 & 44 \\
\hline Virología & 4 & 3 & 3 & 3 & 2 & 4 & 2 & 6 & 3 & 2 & 32 \\
\hline Producción & 2 & 3 & 3 & 3 & 2 & 4 & 1 & 4 & 3 & 1 & 13 \\
\hline Otros & 5 & 12 & 11 & 5 & 12 & 23 & 16 & 18 & 23 & 14 & 139 \\
\hline
\end{tabular}

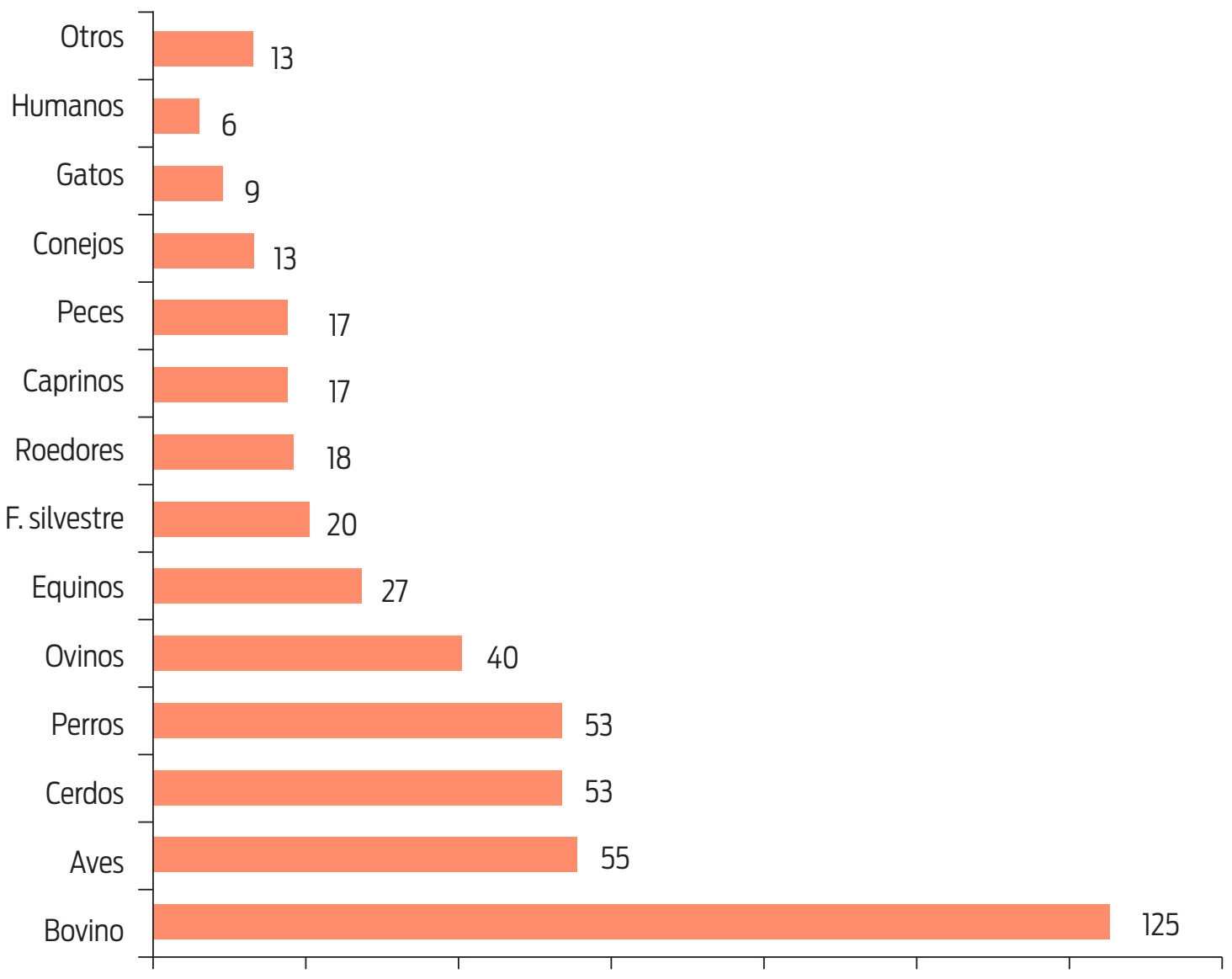

Figura 1. Especies encontradas en los trabajos publicados durante 1989 a 1998 


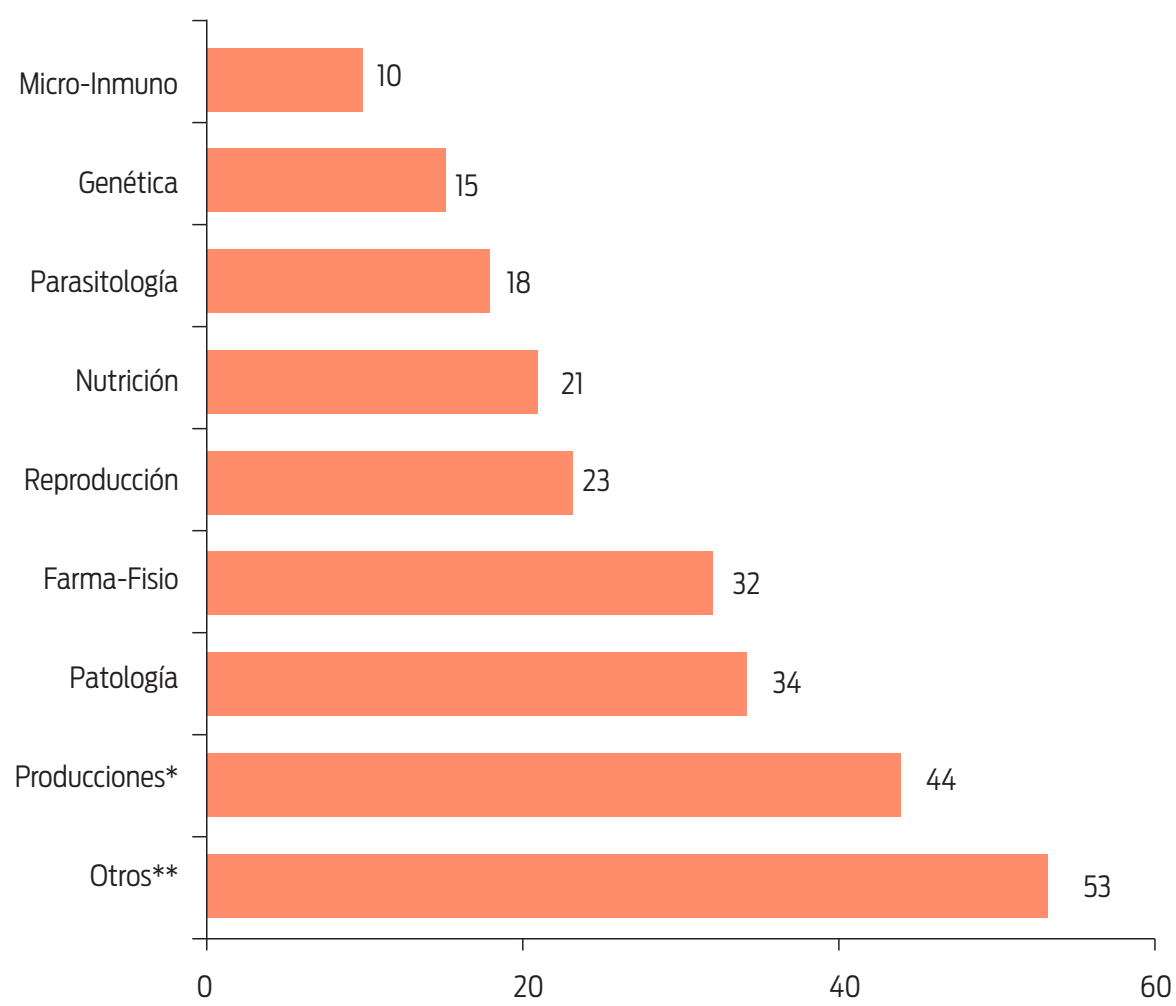

Figura 2. Total de contribuciones de los departamentos de la FMVZ

* Las producciones incluyen bovinos,caprinos, ovinos, cerdos, aves.

**Otros se refiere a los demás departamentos encontrados en la FMVZ

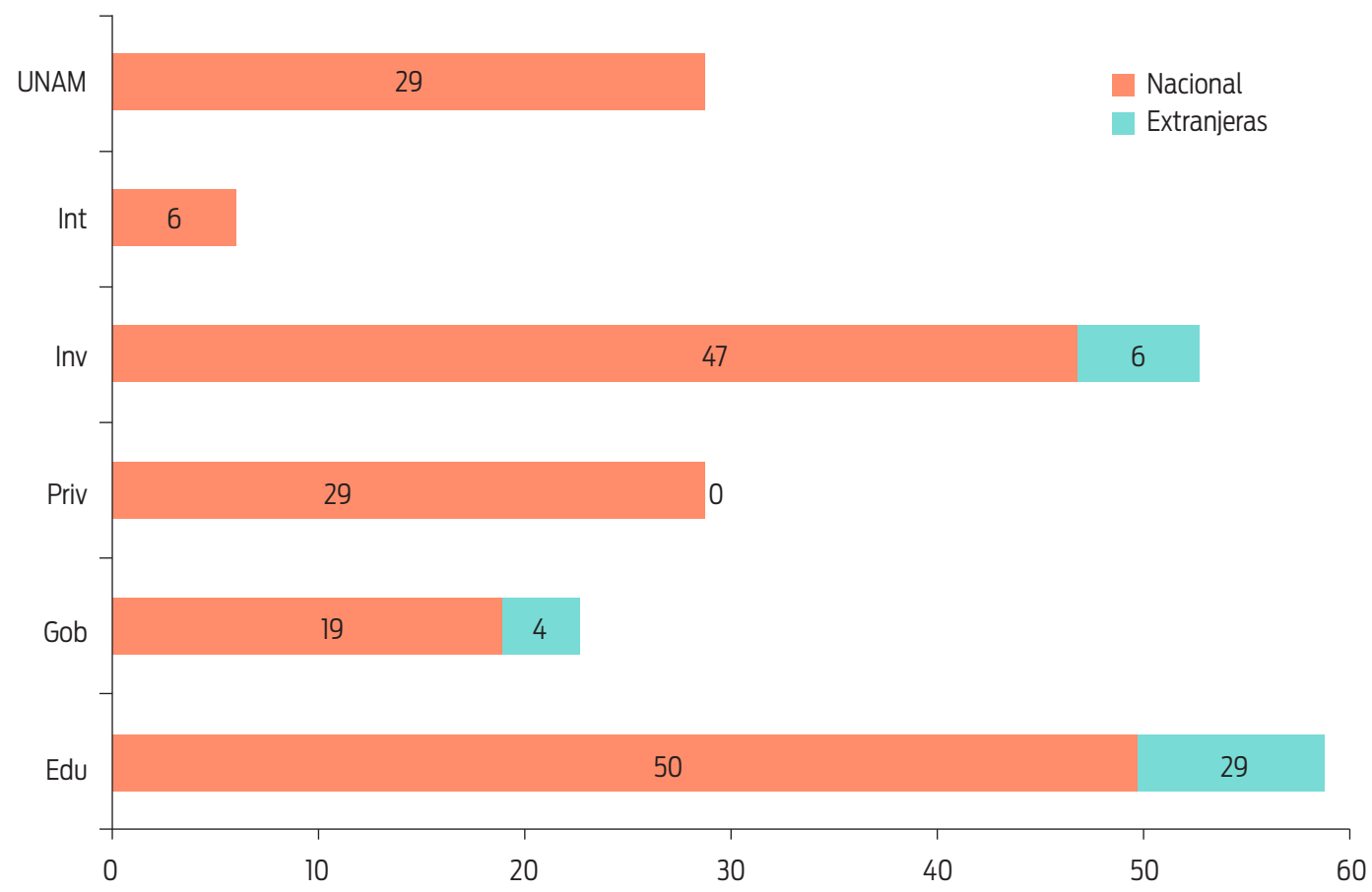

Figura 3. Contribución de la FMVZ en colaboración con otros sectores 


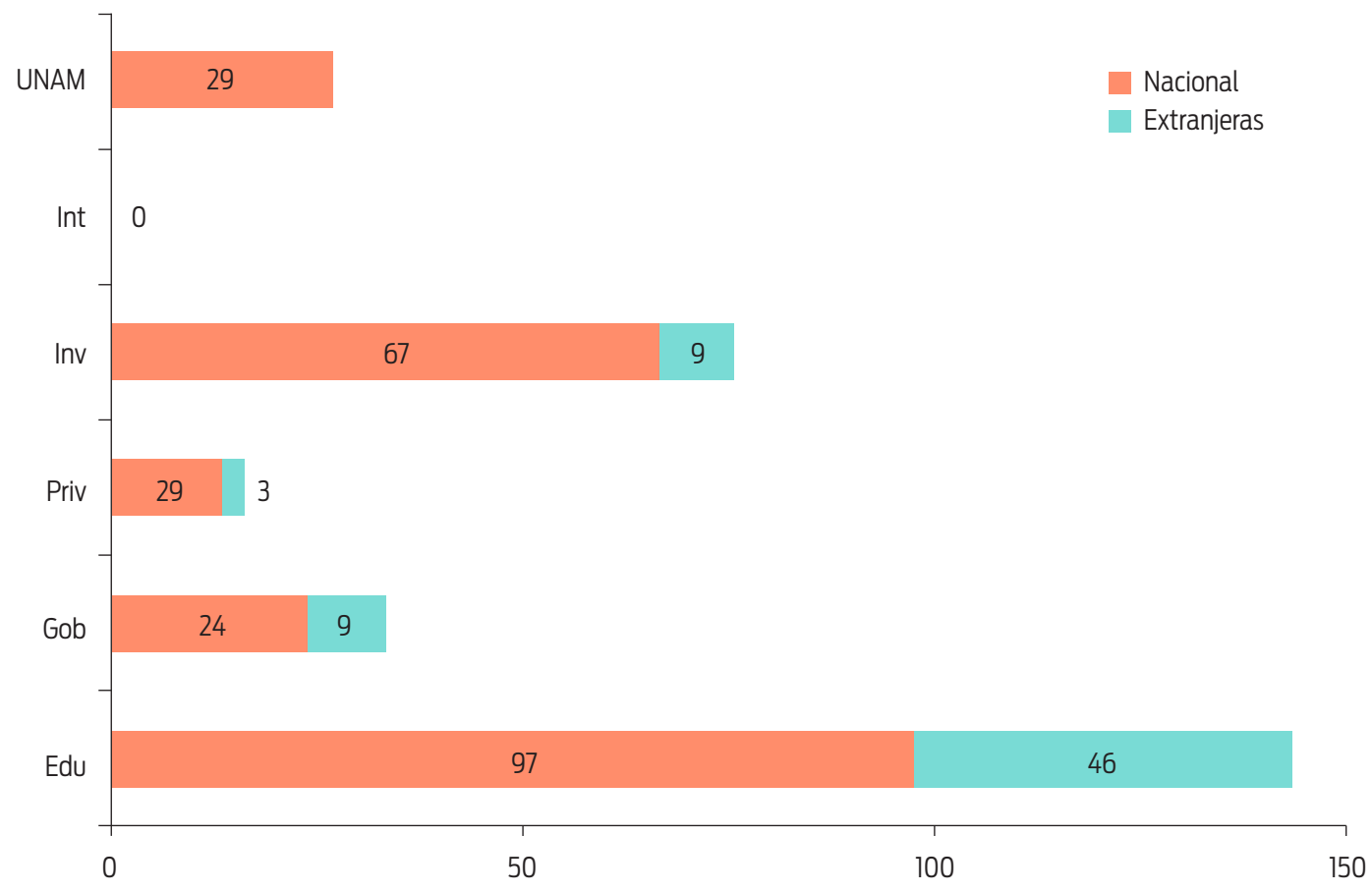

Figura 4. Contribuciones de los distintos sectores sin la colaboración de la FMVZ

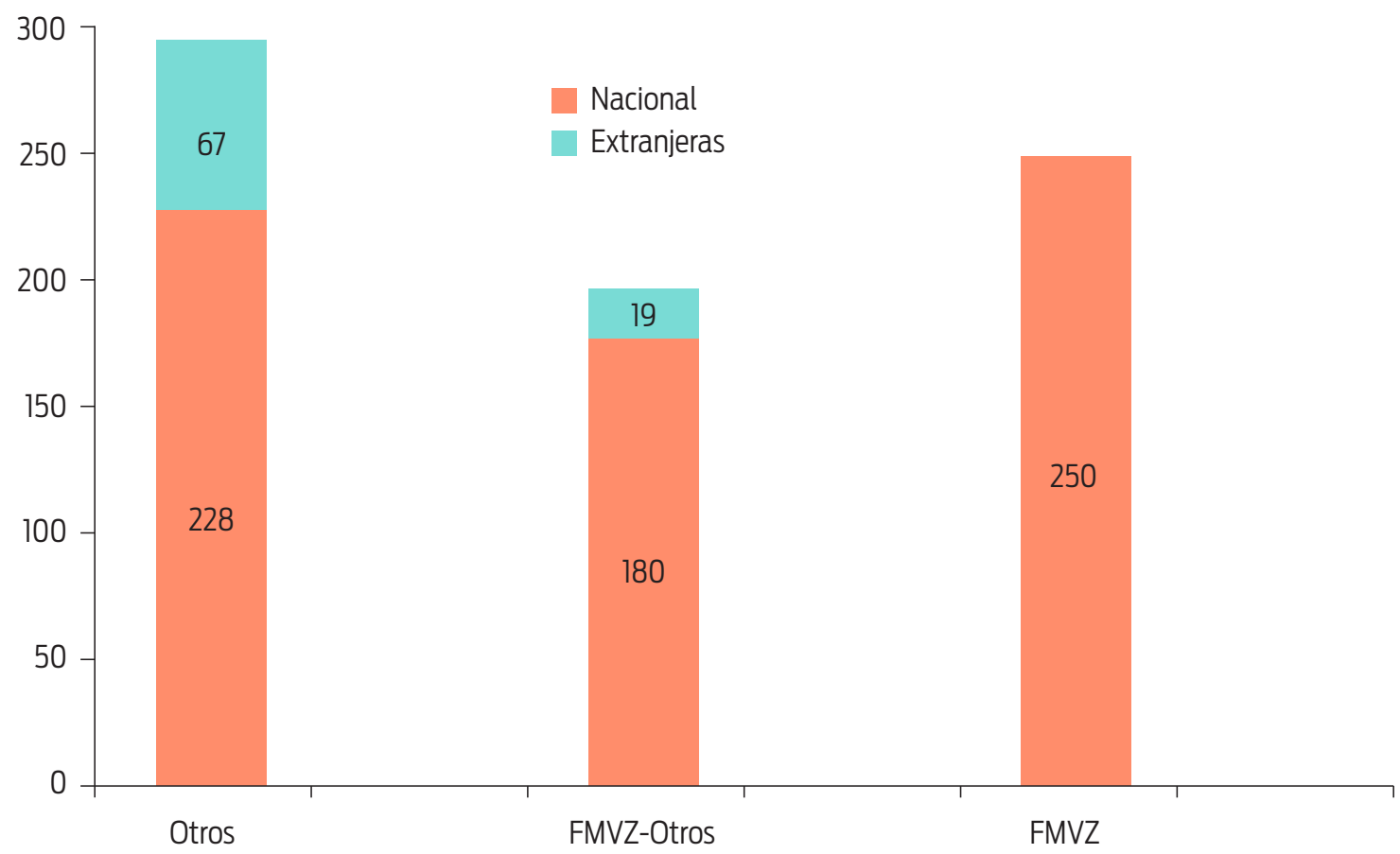

Figura 5. Comparación entre el total de las contribuciones. 


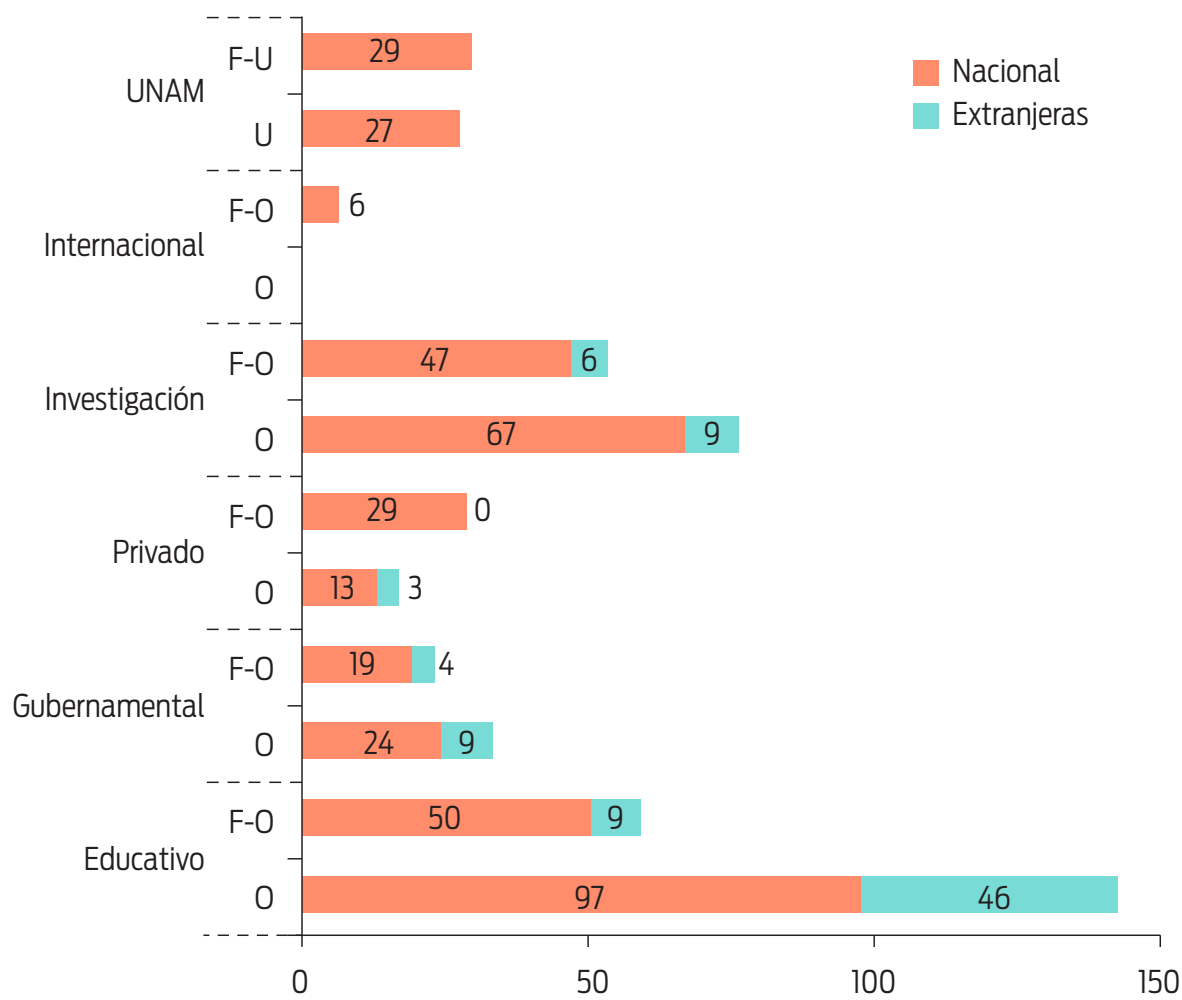

Figura 6. Comparación entre los distintos sectores

$\mathrm{F}-\mathrm{O}=\mathrm{FMVZ}$ y Otros sectores

$\mathrm{O}=$ Otros sectores

$\mathrm{F}-\mathrm{U}=\mathrm{FMVZ}$ y otras dependencias de la UNAM

$\mathrm{U}=$ Otras dependencias de la UNAM

El incremento significativo en el número de trabajos publicados anualmente durante los diez años de 1989 a 1998, analizados en el presente estudio, es similar al observado en otro trabajo para los años 1985 hasta $1988 .^{2}$ El total de contribuciones publicadas por año se incrementó de 31 en 1985 a 56 en 1998. En los primeros cinco años de existencia, Veterinaria México publicó únicamente 103 trabajos correspondientes, en promedio, a unos 21 por año. ${ }^{6}$ Además de su continuidad, la revista ha adquirido un creciente reconocimiento de la comunidad pecuaria, como medio de difusión para la publicación de trabajos originales.

En todos los años analizados, tanto en el presente trabajo como en los dos estudios anteriores, la contribución más importante fue la "Memoria científica" (entre 55\% y 60\%), lo anterior impulsa la difusión de la investigación científica nacional en esta área.

Patología, Reproducción y Parasitología constituyeron las áreas más estudiadas durante los primeros 19 años de existencia de la revista, con porcentajes de cada una de éstas que oscilaban entre el 10 y el 15 del total, lo que representa una importante contribución de estas disciplinas a las investigaciones publicadas por este medio. Nutrición y Virología representaron áreas importantes de investigación durante los primeros años de la revista (10.8\% y 7.9\% del total, respectivamente). ${ }^{6}$ Sin embargo, en el periodo 1989 hasta 1998, éstas representaban 
porcentajes menores, 7.0 y 5, respectivamente. Esto no indica necesariamente que se ha dejado de investigar en estos dos campos. Posiblemente se debe a un incremento en el interés de investigadores de otras áreas para publicar en la revista. Asimismo, Farmacología tuvo poca importancia en los primeros años de publicación (4.4\% del total), pero su participación aumentó a 11.1 \% de 1989 hasta 1998.

Un cambio notable fue un decremento en la participación del departamento de Patología, de $36 \%$ del total de trabajos en los primeros cinco años ${ }^{6}$ a $6.6 \%$ en el presente periodo de estudio.

Este fenómeno se puede explicar, por lo menos parcialmente, debido al incremento en el porcentaje de contribuciones provenientes de otras instituciones. En los primeros 15 años 57.8\% de trabajos emanaron de la propia Facultad, esta cifra disminuyó a 33\% durante 1989 hasta 1998. Los trabajos de diversas instituciones dentro y fuera del país sin la participación de la FMVZ muestran que aun cuando Veterinaria México constituye el órgano de difusión oficial de la FMVZ no publica solamente trabajos propios, sino que existe el interés de otras instituciones nacionales y extranjeras por difundir sus trabajos a través de esta revista. La importante contribución de otras dependencias de la UNAM con y sin la participación de coautores de la propia Facultad sugiere la realización de investigaciones inter y multidisciplinarias dentro del campo de la especialidad.

A lo largo de la trayectoria de Veterinaria México la especie bovina ha sido la más estudiada con alrededor de $23-24 \%$ del total. ${ }^{2,6}$ De igual forma, trabajos sobre aves, porcinos y perros han conservado su importancia para la labor de investigación que se difunde a través de la revista. En el transcurso de los años, la investigación en bovinos ha sido muy productiva, impulsando las diferentes áreas de estudio: Patología, Farmacología-Fisiología, Reproducción, entre otras, o en estudios generales donde se evaluó la solicitud para becas de investigación. 7,8

Los trabajos en coautoría con otras instituciones principalmente del sector educativo y de investigación, en los ámbitos nacional e internacional, indican las buenas relaciones que la FMVZ tiene con ellas. Esto último significa que los científicos de esta institución de primera línea en la investigación en el campo de la medicina veterinaria y zootecnia en el país, están insertos en redes de colaboración que se extiende más allá de las fronteras de la propia comunidad nacional.

Por su parte, los resultados del presente estudio señalan pocos cambios en la fisonomía de las especies y áreas más estudiadas en los trabajos publicados por Veterinaria México, se nota claramente una creciente importancia de la revista como medio de difusión, dentro y fuera de la FMVZ. Un paulatino incremento en los números totales de contribuciones publicadas anualmente más una mayor participación de autores adscritos a otras instituciones apunta hacia una consolidación de Veterinaria México como medio de difusión de la investigación que se realiza principalmente en el país. Como se ha aseverado anteriormente, algunas revistas latinoamericanas han desempeñado un papel significativo como vía de expresión y comunicación de la comunidad científica, contribuyendo así en la definición e impulso de líneas de investigación. ${ }^{9}$ Los resultados del presente análisis sugieren que la revista Veterinaria México es una de ellas. 


\section{Referencias}

1. Vessuri H. Estrategia de valoración de las revistas científicas latinoamericanas. En: Cetto AM, Kai-lnge H, editores. Publicaciones científicas en América Latina. México: International Council of Scientific Unions, Organización de las Naciones Unidas para la Educación, Ciencia y la Cultura, Universidad Nacional Autónoma de México, Academia de la Investigación Científica, Fondo de Cultura Económica; 1995. p. 200-10.

2. Martínez FN. Trayectoria y desarrollo de la revista Veterinaria México a partir de 1985 y su comparación de revistas latinoamericanas afines (tesis de licenciatura). México: Facultad de Medicina Veterinaria y Zootecnia, UNAM; 1995.

3. Facultad de Medicina Veterinaria y Zootecnia. Revista Veterinaria México. México: Facultad de Medicina Veterinaria y Zootecnia, Universidad Nacional Autónoma de México; 1999.

4. Publicaciones en línea de la UNAM. Revista Veterinaria México [citada el 4 de octubre de 1999]. Disponible en: http:// www.unam.mx/cgi-bin/Fomed/ Publica/publil.pl

5. Bonilla M, Pérez-Angón MA. Revistas mexicanas de investigación científica y tecnología. Interciencia. 1999;24:102-6.

6. Pérez HL. Análisis del desarrollo y contenido de los primeros quince volúmenes de la revista Veterinaria-México (tesis de licenciatura). México: Facultad de Medicina Veterinaria y Zootecnia, UNAM; 1988.

7. Mirande A, Galina CS, Russell JM, Navarro-Fierro R. Research in animal reproduction: an analysis of the contribution made by Latin America. Theriogenology. 1987;28:121-7.

8. Riveroll GJ. Perfil de la investigación de los becarios de la Fundación Internacional para la Ciencia en Medicina Veterinaria (tesis de licenciatura). México: Facultad de Medicina Veterinaria y Zootecnia, UNAM; 1997.

9. Cetto AM. Revistas latinoamericanas: un grano de arena a la ciencia universal. Microbiología SEM. 1996;12:647-50. 